\title{
Le don, histoire du concept, évolution des pratiques
}

Thèse de doctorat en philosophie de l'université Paris-Ouest-Nanterre La Défense, sous la direction de Didier Deleule, décembre 2008.

\section{François Athané}

\section{(2) OpenEdition}

Journals

Édition électronique

URL : https://journals.openedition.org/cem/11134

DOI : $10.4000 /$ cem. 11134

ISSN : 1954-3093

Éditeur

Centre d'études médiévales Saint-Germain d'Auxerre

Édition imprimée

Date de publication : 15 août 2009

Pagination : 369-374

ISSN : $1623-5770$

\section{Référence électronique}

François Athané, «Le don, histoire du concept, évolution des pratiques », Bulletin du centre d'études médiévales d'Auxerre | BUCEMA [En ligne], 13 | 2009, mis en ligne le 15 septembre 2009, consulté le 22 septembre 2022. URL : http://journals.openedition.org/cem/11134 ; DOI : https://doi.org/10.4000/ cem. 11134

Ce document a été généré automatiquement le 22 septembre 2022.

\section{c) (†)(2)}

Creative Commons - Attribution - Pas d'Utilisation Commerciale - Partage dans les Mêmes Conditions 4.0 International - CC BY-NC-SA 4.0

https://creativecommons.org/licenses/by-nc-sa/4.0/ 


\section{Le don, histoire du concept, évolution des pratiques}

Thèse de doctorat en philosophie de l'université Paris-Ouest-Nanterre La Défense, sous la direction de Didier Deleule, décembre 2008.

François Athané

Le premier chapitre de cette thèse étudie la notion de don établie en anthropologie et en sociologie par l'Essai sur le don de Marcel Mauss ${ }^{1}$. Il a semblé utile d'examiner en détail les faits sociaux et culturels dont parle Mauss : le potlatch, cette fameuse "guerre des cadeaux » des Amérindiens de la côte nord-ouest du Canada, le kula des archipels Trobriand, à l'est de la Nouvelle-Guinée, le hau des Maoris de Nouvelle-Zélande. La thèse centrale de l'anthropologie de Mauss est que dons et cadeaux constituent la forme la plus ancienne de l'économie : à ce titre, ils sont universels, ayant précédé le troc et le marché. Mais cette position souffre d'une absence de définition précise de ce qu'est un don. Par exemple, il arrive que Mauss nomme «dons » des prestations, qui, après un examen attentif des sources, s'avèrent en réalité dues, parce que leur non effectuation peut conduire à des saisies sur les biens, voire, selon telle page de l'Essai sur le don, à l'esclavage pour dettes. Or, il semble que de telles prestations, en tant qu'elles sont exigibles, méritent le nom de dettes, de tributs ou de taxes, plutôt que de dons.

Lélucidation de ces difficultés conceptuelles dans le texte de Mauss passe par la prise en compte du contexte politique de l'élaboration de l'Essai sur le don. Les conclusions de cet écrit font au demeurant directement référence à la 
conjoncture économique et sociale des sociétés européennes d'après la première guerre mondiale. La publication par Marcel Fournier des Écrits politiques de Mauss ${ }^{2}$ permet désormais de mieux comprendre les préoccupations sociales de l'Essai sur le don, notamment cette idée, maintes fois exprimée par Mauss, que le don, l'échange et le commerce sont les seuls antidotes possibles à la guerre, à l'isolement et à la stagnation. Les crises économiques et monétaires dans l'Europe d'après-guerre, les conséquences désastreuses du Traité de Versailles sur l'économie européenne et les relations internationales ont conduit Mauss à chercher dans les sociétés archaïques les fondements économiques et symboliques par lesquels peut s'engager entre les peuples une paix durable, elle-même nécessaire à la prospérité économique. L'idée que le don est la première forme d'alliance, de solidarité et de pacification sociales est en somme étroitement liée, chez Mauss, à son analyse de la guerre entre les nations européennes au début du XXe siècle, et de la guerre civile dans la Russie d'après 1917, ainsi qu'aux engagements de l'auteur pour des politiques économiques plus solidaires, à orientation socialiste. Mais cette convergence supposée, entre ce que nous apprennent les économies archaïques et les préoccupations politiques de l'auteur, tend à produire quelque confusion, dans les conclusions de l'essai de Mauss, entre des réalités fort différentes: le don d'une part, et d'autre part le crédit voire les assurances sociales mutuelles ou les coopératives.

Le deuxième moment de la thèse consiste à montrer que ce flou conceptuel sur le don, et cette absence d'une distinction précise entre le don, le crédit, l'échange ou le tribut, ont perduré dans les écrits des sociologues et philosophes ultérieurs. Le propos est alors de marquer les apports et les limites des travaux de divers auteurs, notamment René Maunier, disciple méconnu de Mauss qui tenta d'appliquer la problématique maussienne du don à l'étude des échanges rituels en Afrique du Nord ${ }^{3}$, mais aussi Claude Lévi-Strauss ${ }^{4}$, Pierre Bourdieu ${ }^{5}$ et Jacques Derrida ${ }^{6}$. Une lecture détaillée de chacun de ces auteurs montre que leurs théorisations, aussi variées soient-elles, achoppent sur une même difficulté. Il s'agit de la distinction entre les transferts exigibles et ceux qui ne le sont pas, c'est-à-dire entre, d'une part, les transferts dont la non effectuation peut entraîner l'exercice de la violence légitime (coutumière ou étatique), transferts qui dès lors peuvent 
être considérés comme exigibles, et, d'autre part, les transferts de biens qui ne sont pas exigibles, parce que leur non effectuation ne débouche pas sur le recours possible à la violence légitime.

Le troisième chapitre essaie de constituer ce qui a fait défaut aux chercheurs précédents : une typologie générale de toutes les formes possibles de transferts de biens entre les humains. Ce sont alors principalement les travaux d'anthropologie économique d'Alain Testart qui sont sollicités et commentés ${ }^{7}$. Le propos consiste à montrer que l'on a jusqu'à présent décrit les mouvements des biens à l'intérieur des sociétés : ce que l'on peut appeler la cinétique des transferts, mais que l'on s'est trop peu préoccupé des droits et devoirs associés aux mouvements de biens et aux prestations de services, ce que l'on peut appeler la déontique des transferts. Or, c'est seulement en tenant compte de ces questions de droits et de devoirs associés au mouvement des biens que l'on peut différencier le don de l'échange, l'échange de l'impôt ou de l'amende; et, au sein des échanges, cette sorte très particulière d'échanges que sont les marchés et les échanges marchands. Il a en effet semblé, à l'issue de bien des lectures, que les définitions que l'on donne généralement du «marché » - et plus encore de «l'économie de marché »- sont très variables. On arrive dès lors aux définitions suivantes :

-le don est un transfert non exigible sans contrepartie exigible ;

-l'échange est l'ensemble formé par deux transferts dont chacun est la contrepartie exigible de l'autre ;

-il existe une forme de transfert pour laquelle on ne dispose pas de nom générique en français : il s'agit du transfert exigible sans contrepartie exigible. Le tribut, l'impôt, l'amende, le dédommagement ressortissent à ces transferts du troisième type, ou t3t, pour reprendre la terminologie d'Alain Testart ;

- enfin, il existe des transferts, qui, bien que non exigibles, sont néanmoins exigés et obtenus par un usage illégitime de la force : on parlera dans ce cas de prédation.

Quant au marché, c'est une sous-espèce de l'échange, qui a pour particularité, par rapport à d'autres formes de l'échange, de ne pas requérir entre les partenaires échangistes de lien social préalable à l'acte d'échange. Sur un marché, vendeur et acheteur peuvent être des inconnus l'un pour l'autre, critère par lequel on peut clairement 
saisir que la plupart des échanges décrits par Mauss en son essai ne sont pas des échanges marchands. Par exemple, dans le kula des îles Trobriand, les partenaires ont entre eux des relations sociales qui préexistent à l'acte d'échanger : on parlera en ce cas d'échange entre amis. La particularité du marché est de pouvoir constituer des échanges entre des individus ou des groupes qui n'ont pas de liens sociaux ou d'interconnaissance préalable à l'acte d'échange. Ce qui implique, selon toute vraisemblance, l'existence d'une forme ou d'une autre de l'État comme préalable nécessaire à la généralisation des échanges marchands. En effet, seuls des dispositifs de type étatique, avec un haut degré de concentration des moyens de la contrainte physique, peuvent garantir l'exigibilité de la contrepartie dans l'échange, en l'absence de liens d'interconnaissance entre les partenaires échangistes.

Cette typologie des transferts de biens et prestations de services, le quatrième chapitre s'efforce d'en montrer la pertinence, à propos d'un cas particulier: l'histoire économique, politique et religieuse du Moyen Âge. On tente d'y montrer combien sont incertaines, aux époques mérovingiennes et carolingiennes, les frontières entre le don, l'impôt et la prédation pure et simple. Reprenant certaines idées formulées par Marc Bloch 8, Georges Duby ${ }^{9}$ ou Norbert Elias ${ }^{10}$, on essaie alors d'indiquer comment la construction des seigneuries puis des États monarchiques est étroitement dépendante, dans l'Europe occidentale du Moyen Âge, d'une idéologie du don telle que les seigneurs et les rois entretiennent par les dons des réseaux de fidélités, qui progressivement se muent en administrations et en bureaucraties. Mais ce qui se présente alors sous le vocabulaire du don, de la largesse et de la générosité relève en fait de nécessités politiques auxquelles les puissants devaient sacrifier, à moins de perdre leurs appuis politiques et militaires : ce sont les "générosités nécessaires » dont parle Georges Duby. Symétriquement, il est fréquent qu'une rhétorique de l'échange - par exemple, le «prix de la paix » exigé par les seigneurs auprès des marchands qui passaient sur leurs terres - permette de masquer le prélèvement fiscal sans contrepartie. En somme, tout se passe comme si la vérité des rapports de forces et des enjeux de domination cherchait constamment à se dissimuler sous le vocabulaire du don et de l'échange.

L'importance du don dans l'économie et l'idéologie de l'Église catholique est également analysée, à partir des 
travaux d'Eliana Magnani ${ }^{11}$, de Dominique Iogna-Prat ${ }^{12}$ et d'Anita Guerreau-Jalabert ${ }^{13}$. Le vocabulaire et la rhétorique du don permettent alors de désigner, et de masquer à la fois, de véritables échanges contractuels entre les laïcs fortunés et les institutions ecclésiastiques. Des prestations en biens ou en terres fiscales ont alors pour contreparties dues des offices célébrés pro remedio animae. On décèle ainsi, sous une rhétorique de la gratuité et de la générosité, un système de droits et de devoirs réciproques qui relève de l'échange de biens matériels en contrepartie de prestations de salut.

On insiste ensuite sur le don et la charité comme traitement de la souffrance sociale et de la misère durant l'Ancien Régime ; enfin sur le basculement que représentent, à cet égard, la révolution française et les luttes sociales des XIX et $\mathrm{XX}^{\mathrm{e}}$ siècles, dans la mesure où elles ont permis l'atténuation progressive du don dans le traitement social de la misère. En effet, les allocations de subsistance, le droit à l'éducation, à la santé ou autres droits sociaux ne sont structurellement pas des dons, mais des dus sans contrepartie exigible - quelle que soit la trompeuse rhétorique du don persistant dans les discours sur 《l'assistanat», «l'État providence », etc. De telles prestations sociales sont dès lors à ranger dans la catégorie des transferts du troisième type. On assiste ainsi, avec la genèse et le développement de l'État social, à ce que l'on peut appeler un redoublement de l'exigibilité. Car ce n'est plus seulement le pouvoir politique qui peut exiger des particuliers un transfert sans contrepartie (l'impôt); désormais ce sont aussi les particuliers qui peuvent, selon la législation sociale en vigueur, exiger de l'État une prestation sans contrepartie.

Le dernier chapitre revient sur l'idée, déjà présente chez Mauss, que le don serait universel. Cette idée que tous les groupes humains pratiquent le don suppose qu'une comparaison soit menée avec d'autres espèces animales: pourquoi donc les autres animaux ne pratiqueraient-ils pas eux-mêmes le don ? Il s'agit alors d'examiner la très grande diversité des formes de transferts de ressources chez les autres animaux. À l'issue de cette étude, il est montré en quoi il y a bel et bien une différence entre les transferts de biens chez les humains, d'une part, et les transferts de ressources chez les autres espèces animales, qu'il s'agisse des fourmis ou des chimpanzés. Précisément, cette différence tient en ceci que les transferts de biens, chez les 
humains, ne constituent pas seulement une cinétique des ressources; ils sont dotés de structures déontiques, avec des droits et des devoirs associés à des mouvements de biens.

L'enjeu est alors d'indiquer quelles sont les causes, qui, dans la sélection naturelle ayant produit l'espèce humaine, ont nécessairement induit l'importance cruciale du don chez tous les êtres humains. Il s'agit notamment du fait que les humains en bas âge ont vitalement besoin des transferts qui leur proviennent des adultes, alors même que pendant une durée assez longue ces mêmes enfants sont dans l'impossibilité de contracter avec les adultes. Les flux de ressources des adultes vers les nourrissons sont alors nécessairement des dons, et non pas des échanges.

Ce travail s'achève par une réflexion sur les dévastations que cause, aujourd'hui même, l'absence du don. Le propos porte alors sur ces êtres qui, par-dessus tout, ont absolument besoin qu'on leur donne : les enfants, et sur ce qui leur advient lorsqu'on ne leur donne pas, ou pas assez. Le fait que des dizaines de millions de filles du monde contemporain soient tuées par infanticide actif ou par maltraitance, ou que des enfants, non moins nombreux, soient, chaque année, vendus aux fins de travail ou d'esclavage prostitutionnel, cela peut et doit s'analyser comme résultant de conditions sociales, économiques et culturelles spécifiques qui induisent l'impossibilité ou le refus du don entre les générations ${ }^{14}$.

\section{NOTES}

1. M. MAUSS, «Essai sur le don. Forme et raison de l'échange dans les sociétés archaïques ", Année sociologique, 1 (1923-1924, nouv. série), p. 30-186, repris dans M. MAUSS, Sociologie et anthropologie, intro. de C. LÉVI-STRAUSS, Paris, 1950, p. 143-279.

2. M. MAUSS, Écrits politiques, textes réunis et présentés par M. FOURNIER, Paris, 1997.

3. R. MAUNIER, "Recherches sur les échanges rituels en Afrique du Nord ", Année sociologique, 2 (1924-1925, nouv. série), p. 11-97, repris avec des variantes dans Coutumes algériennes, Paris, 1935, et dans Recherches sur les échanges rituels en Afrique du Nord, présentation et notes d'A. MAHÉ, SaintDenis, 1998.

4. C. LÉVI-STRAUSS, "Introduction à l'œuvre de Marcel Mauss", in M. MAUSS, Sociologie et anthropologie..., op. cit., p. IX-LII. 
5. P. B OURDIEU, Esquisse d'une théorie de la pratique, précédée de Trois études d'ethnologie kabyle, Genève, 1972 (rééd. Paris, 2000) ; ID., Le Sens pratique, Paris, 1980.

6. J. DERRIDA, Donner le temps. 1 : la fausse monnaie, Paris, 1991.

7. A. TESTART, Des Dons et des dieux, Paris, 1993 (rééd. 2006) ; ID., Critique du don, Paris, 2007.

8. M. BLoch, Rois et serfs, un chapitre d'histoire capétienne, Paris, 1920, rééd. Rois et serfs et autres écrits sur le servage, postface de D. BARTHÉLEMY, Paris, 1996 ; ID., La société féodale, Paris, 1940, 2 vol. (rééd. en un volume, 1968).

9. G. Duby, Guerriers et paysans, VII ${ }^{\mathrm{e}}-\mathrm{XII} \mathrm{e}^{\mathrm{e}}$ siècle. Premier essor de l'économie européenne, Paris, 1973.

10. N. Elias, Über den Prozess der Zivilisation, t. 2 (Wandlungen der Gesselschaft), Francfort-sur-leMain, 1969, trad. fr. P. KAMNITZER, La Dynamique de l'Occident, Paris, 1975 (rééd. Paris, s. d.).

11. E. MAGNANI, "Le don au Moyen Âge: pratique sociale et représentations. Perspectives de recherche», Bulletin du Centre d'études médiévales d'Auxerre, 4 (2000), p.62-79; EAD., «Les médiévistes et le don. Avant et après la théorie maussienne ", inE. MAGNANI (dir.) Don et sciences sociales. Théories et pratiques croisées, Dijon, 2007, p. 15-28.

12. D. IOGNA-PRAT, Ordonner et exclure. Cluny et la société chrétienne face à l'hérésie, au judaïsme et l'islam (1000-1150), Paris, 1998 (2 éd. corrigée, Paris, 2000).

13. A. GUeRREAU-JALABERT, "Caritas y don en la sociedad medieval occidental ", Hispania. Revista Espanola de Historia, 60/1/204 (2000), p. 27-62. Je remercie Eliana Magnani de m'avoir communiqué la version française de ce texte.

14. Je remercie Eliana Magnani d'avoir bien voulu prendre place au jury de ma thèse.

\section{INDEX}

Mots-clés : don 